\title{
EDIFICATE
}

I Congreso de Escuelas de Edificación y Arquitectura Técnica de España

València, 4 y 5 de noviembre de 2021

Escuela Técnica Superior de Ingeniería de Edificación

Universitat Politècnica de València

Doi: https://doi.org/10.4995/EDIFICATE2021.2021.13300

\section{La dificultad como oportunidad de cambio: mejoras docentes tras una experiencia íntegramente online}

\section{In difficulty lies opportunity: teaching improvements after an entirely online experience}

\author{
Lucía Reig-Cerdá a , María José Ruá-Aguilar a , Ángel Pitarch-Roig a y Joaquín \\ Martínez-Moyá b \\ a Universitat Jaume I, Departamento de Ingeniería Mecánica y Construcción, Ireig@uji.es, rua@uji.es \\ pitarch@uji.es, b Universitat Jaume I, Departamento de Ingeniería de Sistemas Industriales y Diseño, \\ jomoya@uji.es
}

\section{Abstract}

The global pandemic of Covid-19 forced to all educational levels to adapt in record time to an entirely online teaching. It was necessary to adapt content, materials and methodologies, maintaining the quality of teaching. Despite the fact that universities had various tools, such as their virtual platforms, which have improved student-teacher interaction and facilitate the management of resources and content, they were not prepared for such a drastic and significant change. However, the university community proved to be quickly adapted and moved on, and the update process entailed an opportunity to change towards a new, more active and student-centered teaching model. This communication presents the practical experience of a subject of construction materials of the degree in Technical Architecture of the Universitat Jaume I. It describes how the teaching was adapted during confinement and afterwards, how the experience improved the subject in a face-to-face or hybrid teaching model. The training in digital skills of the teaching staff, the new possibilities of communication with the students, or the new habits acquired have made it possible to improve the teaching-learning process, developing various activities to support face-to-face tasks, more attractive, interactive and motivating, which help students to improve their skills and learning outcomes.

Keywords: teaching methodologies, virtual resources, flexible learning, autonomous learning, H5P tools. 


\section{Resumen}

La pandemia mundial de la Covid-19 obligó a adaptar en tiempo récord una docencia íntegramente online en todos los niveles educativos. Fue necesario adaptar contenidos, materiales y metodologías, evitando perjudicar a la calidad de la enseñanza. Pese a que las universidades contaban con diversas herramientas, como sus plataformas virtuales, que mejoran la interacción alumno - profesor y facilitan la gestión de recursos, no estaban preparadas para un cambio tan drástico y significativo. No obstante, la comunidad universitaria se adaptó rápidamente y siguió adelante, actualizándose, lo que derivaría en una oportunidad de cambio hacia un nuevo modelo de enseñanza más activo y centrado en el estudiantado. Esta comunicación presenta la experiencia práctica de una asignatura de materiales de construcción del grado en Arquitectura Técnica de la Universitat Jaume I. Se describe cómo se adaptó la docencia durante el confinamiento para, posteriormente, incorporar mejoras en el modelo presencial o híbrido. La formación en competencias digitales del profesorado, las nuevas posibilidades de comunicación con el alumnado, o los nuevos hábitos adquiridos han permitido mejorar el proceso de enseñanzaaprendizaje, desarrollando diversas actividades de apoyo a las tareas presenciales, más atractivas, interactivas y motivadoras, que ayuden al alumnado a mejorar sus competencias y resultados de aprendizaje.

Palabras clave: metodologías docentes, recursos virtuales, flexibilidad en el aprendizaje, aprendizaje autónomo, herramientas H5P. 


\section{Introducción}

Los cursos académicos 2019-2020 y 2020-2021 han estado fuertemente condicionados por la pandemia mundial de la Covid-19. En marzo de 2020, se decretó en España un confinamiento obligatorio para la población, que afectó a todos los sectores de la Sociedad. La enseñanza no fue una excepción y, todos los niveles educativos tuvieron que adaptarse a las nuevas condiciones. Durante estos dos cursos se ha experimentado desde un confinamiento total, con una enseñanza totalmente virtual, a periodos de docenciaaprendizaje presenciales (guardando las preceptivas condiciones de seguridad: aforos, distancias, mascarillas...) y, en algunos casos, situaciones híbridas.

Docentes y alumnado han tenido que responder a las circunstancias cambiantes, siendo especialmente duro el periodo en el que, bajo una situación de emergencia, la docencia fue $100 \%$ online. Dicho periodo puso a prueba la capacidad de resiliencia a nivel personal y fue una oportunidad para testar el potencial de las herramientas para una enseñanza a distancia. A nivel universitario, las plataformas virtuales facilitaron mucho dicha adaptación, permitiendo compartir contenidos, impartir clases por videoconferencia y/o evaluar las asignaturas. A diferencia de lo que ocurrió en otros ámbitos y sectores de la sociedad, dichas herramientas permitieron mantener y adaptar la actividad universitaria. No obstante, tal como apuntan Adell y Andrés (Adell y Andrés, 2020), en aquel momento pasamos rápidamente a una educación en línea de emergencia, diferente de una educación a distancia, que requiere pedagogía y planificación.

La Universitat Jaume I, como la mayoría de las universidades públicas españolas, siempre ha apostado por una docencia presencial. Dentro de ella, una característica diferencial del grado en Arquitectura Técnica es que incorporó desde sus inicios la docencia por proyectos (Reig et al., 2017). Bajo la denominación de Proyecto Dirigido el alumnado, organizado por equipos, desarrolla un trabajo sobre un edificio real, lo que les permite tanto aplicar los conocimientos teóricos adquiridos en las diferentes asignaturas de cada curso, como trabajar otras competencias, como el trabajo en equipo, el análisis de documentación, la síntesis de información o la presentación de resultados ante un tribunal formado por profesores de las diferentes asignaturas (Reig et al., 2017; Martínez-Moya et al., 2020). Por tanto, dicha metodología eminentemente práctica, requiere interacción, tanto entre estudiantes para llevar a cabo el desarrollo del trabajo, como con el profesorado, que realiza su seguimiento. Fue necesario desarrollar nuevos hábitos, nuevas formas de comunicación y relacionarse y, pese a que la presencialidad permite un contacto personal que es difícil de suplir desde las pantallas, el uso de determinadas herramientas permitió comunicarse y trabajar de manera remota, síncrona y/o asíncrona, lo que permitió alcanzar las habilidades y competencias previstas, aunque siguiendo procedimientos distintos a los tradicionalmente empleados.

Tras la experiencia vivida, (Pitarch-Roig et al., 2020) era necesario reflexionar y aprender de lo vivido, tratando de optimizar el esfuerzo realizado para mejorar el proceso de enseñanzaaprendizaje. 


\section{Objetivos}

El objetivo de la presente comunicación es describir cómo se ha modificado la docencia en la asignatura 'Materiales de Construcción III' (Mdc3) del grado en Arquitectura Técnica de la Universitat Jaume I el curso 2020-21, tras su adaptación de emergencia para una docencia totalmente online en periodo de confinamiento.

\section{Metodología para mejorar la docencia de la asignatura Materiales de Construcción III}

La Figura 1 recoge la metodología seguida para mejorar el proceso enseñanza-aprendizaje en la asignatura Mdc3 del grado en Arquitectura Técnica de la Universitat Jaume I de Castellón. La presente comunicación se ha organizado en los mismos apartados, describiendo en primer lugar como se adaptó la docencia online durante la situación de emergencia sanitaria, para posteriormente reflexionar sobre los cambios que implicó, las oportunidades para rediseñar el proceso de docencia-aprendizaje universitarios y las necesidades de formación que ello implicaba. Se describirán la formación y apoyo recibidos, tanto durante la adaptación de emergencia a una docencia $100 \%$ online, como para el posterior desarrollo de una docencia presencial y/o híbrida. Se recogerán los cambios introducidos en la asignatura que, utilizando herramientas virtuales como soporte a la docencia presencial, facilitan la tarea docente y hacen más atractiva y dinámica la participación del alumnado, promoviendo también su capacidad de aprendizaje autónomo. Finalmente, se realizarán propuestas que permitan continuar mejorando la asignatura.

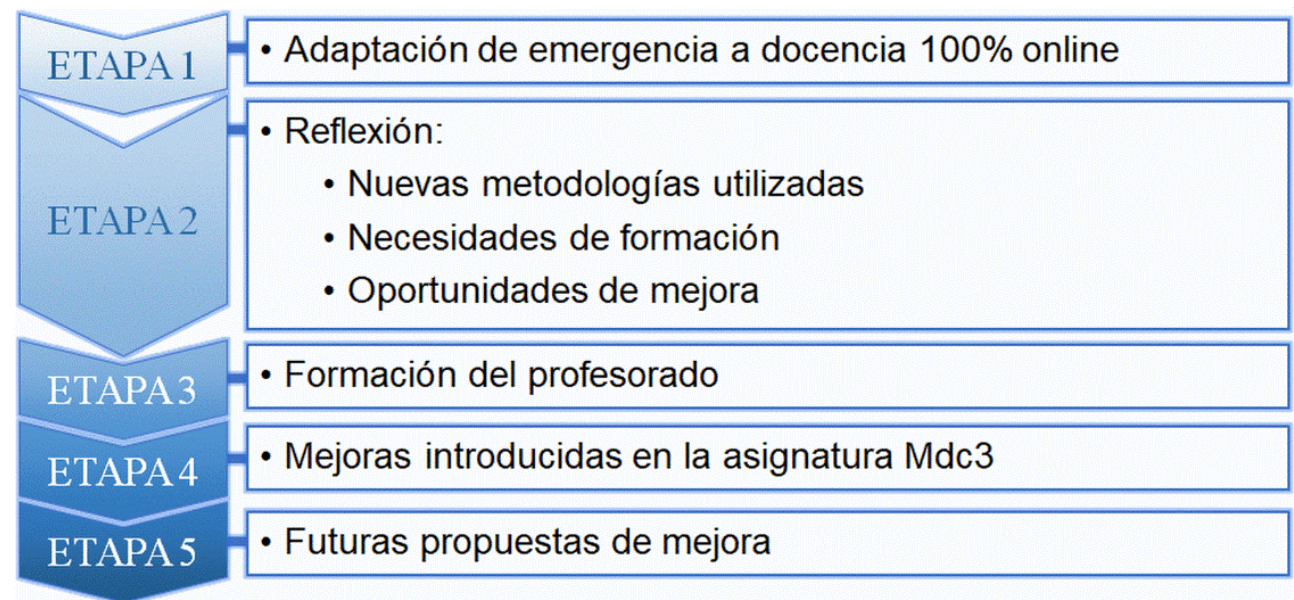

Fig. 1 Metodología seguida para mejorar el proceso enseñanza-aprendizaje en la asignatura Mdc3 


\section{Resultados. Mejora de la asignatura Materiales de Construcción III del grado en Arquitectura Técnica de la Universitat Jaume I}

En el siguiente apartado se describirá la asignatura objeto de mejora, para posteriormente describir cómo se adaptó su docencia durante el periodo de confinamiento, analizar las posibilidades de mejora que de ello derivaron, las necesidades de formación del profesorado, y las mejoras introducidas al volver a impartir la asignatura de forma presencial. Finalmente, tras reflexionar sobre este proceso de cambio, se realizarán nuevas propuestas de mejora para la asignatura, extrapolables a otras asignaturas del ámbito de la edificación.

\subsection{La asignatura}

Tal como recoge la Tabla 1, la asignatura Mdc3 impartida en el grado en Arquitectura Técnica de la Universitat Jaume I es obligatoria de 6 créditos, impartida en el segundo semestre de segundo curso. Se ha elegido esta asignatura para describir el proceso de modificación de la enseñanza-aprendizaje porque, cuando se decretó el estado de alarma en marzo de 2020, estaba siendo impartida de forma presencial y tuvo que ser adaptada de urgencia para una docencia totalmente online.

Tabla 1. Principales descriptores de la asignatura Mdc3

\begin{tabular}{ccccccc}
\hline Código & Nombre & Curso & Semestre & Créditos & $\begin{array}{c}\text { Horas } \\
\text { presenciales }\end{array}$ & $\begin{array}{c}\text { Horas no } \\
\text { presenciales }\end{array}$ \\
\hline ED0919 & $\begin{array}{c}\text { Materiales de } \\
\text { Construcción III }\end{array}$ & 2 & 2 & 6 & 57 & 93 \\
\hline
\end{tabular}

\subsection{Adaptación a la docencia en línea de emergencia}

La situación sanitaria del momento obligó a adaptar urgentemente la docencia a un modelo totalmente online: había que adaptarse de forma rápida y continuar. Era un nuevo modelo para el que fue necesaria una adaptación inmediata sin precedentes.

Para ello, el apoyo de la universidad fue clave, la cual fue flexible y trató de responder de la mejor forma posible a las necesidades de alumnado y profesorado; el apoyo de compañeros que compartían sus experiencias, dificultades y soluciones; así como del Centre d'Educació i Noves Tecnologies de la Universitat Jaume I (CENT, 2021), quien trató de resolver las diferentes dudas e inquietudes con gran eficiencia y profesionalidad. Así, en tiempo récord se generaron diversas guías de apoyo, procedimientos y mejoras tecnológicas que permitieron adaptar la docencia a las exigencias del momento.

En el caso concreto de Mdc3 se adaptaron las actividades, que tradicionalmente habían sido mayoritariamente presenciales y, tal como recoge la Tabla 2, se diferenció entre tareas que debían realizarse antes de la sesión virtual síncrona (a la hora de la clase), durante o después. 
La dificultad como oportunidad de cambio: mejoras docentes tras una experiencia íntegramente online In difficulty lies opportunity: teaching improvements after an entirely online experience

Tabla 2. Adaptación de actividades la asignatura Mdc3 durante el periodo de confinamiento

\begin{tabular}{|c|c|c|c|}
\hline \multirow{3}{*}{ 옹 } & \multicolumn{3}{|c|}{ REALIZACIÓN } \\
\hline & ANTES & DURANTE & DESPUÉS \\
\hline & \multicolumn{2}{|c|}{ De la sesión Online Síncrona } & \\
\hline 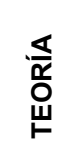 & - & $\begin{array}{l}\text { - Explicación de conocimientos } \\
\text { teóricos mediante Google Meet, } \\
\text { compartiendo presentación con los } \\
\text { alumnos. Y actividades de } \\
\text { autoevaluación de forma síncrona. }\end{array}$ & - \\
\hline
\end{tabular}

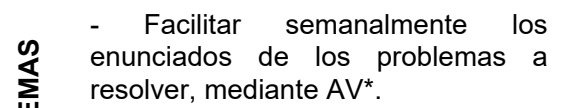

- Alumnado resuelve los problemas de forma autónoma.

- Entregan los problemas mediante tarea creada en AV.
- Resolución de problemas, compartiendo pantalla con los

alumnos mediante Google Meet

- Adaptar la práctica presencial a un formato online, en el que se sustituyeron las actividades de

of laboratorio por videos, fotografías... que les permitiesen trabajar los mismos conocimientos y habilidades. - Facilitar el enunciado de la práctica de laboratorio mediante AV.

- Conexión mediante Google Meet para dudas y aclaraciones - Alumnado completa el enunciado de forma autónoma.

- Entregan mediante tarea creada en AV.
- El alumnado resuelve el cuestionario de autoevaluación de la práctica de laboratorio correspondiente, mediante AV
음 - Desarrollo de forma autónoma por 을 parte del estudiantado.

음 - Directrices por parte de los (丆 profesores sobre como comunicarse 으 entre ellos: trabajando de forma U. colaborativa mediante Google Drive, щ reuniéndose telemáticamente con Google Meet, comunicación por 㩊 teléfono o WhatsApp...
- Tutorías mediante Google Meet para dudas y aclaraciones
- Desarrollo de forma autónoma por parte del estudiantado tras revisión y aclaración de dudas.
- Evaluación del contenido del trabajo desarrollado en Proyecto Dirigido.

- Modelo de examen, facilitado a través del AV. Resolución de dudas mediante tutoría online síncrona por Google Meet.
- Valoración, por parte de un tribunal de la defensa del trabajo desarrollado en Proyecto Dirigido.

- Realización del examen de la asignatura mediante un cuestionario realizado en $\mathrm{AV}$. Con cuestiones tipo test, cuestiones cortas y problemas. Con control del tiempo en cada una de las partes.
- Revisión de la calificación obtenida mediante foro de tutorías privadas del aula virtual, y mediante videoconferencia por Google Meet.

\footnotetext{
* AV: Aula Virtual (plataforma Moodle en la UJI)
} 
La mayor dificultad a la que se vieron sometidos estudiantes y profesores en este periodo fue no tener un contacto personal, lo cual se vio especialmente reflejado en las prácticas de laboratorio y la realización del Proyecto Dirigido, pues éste requería trabajo en equipo para su desarrollo y comunicación con los profesores para su revisión y defensa (Reig et al., 2017; Martínez-Moya et al., 2020). Por otra parte, la realización del examen de forma virtual, con el alumnado en sus casas y frente a un ordenador, fue también todo un reto, tanto para el estudiantado como para el profesorado (Pitarch-Roig et al., 2020). Para los primeros, por la inseguridad que les generaba pensar que no contaban con la presencia física del profesorado (aunque estuviesen conectados por videoconferencia), un posible fallo de conexión a internet, o el hecho de realizar un examen frente a un ordenador, cada uno con los condicionantes de sus propias viviendas. Para los segundos, por el reto que suponía diseñar un examen para su realización completamente online, con las incertidumbres legales sobre las posibles limitaciones para controlar el fraude durante la realización de las pruebas y la dificultad en acotar el tiempo sin limitarlo en exceso...

A pesar de todo, la experiencia vivida demostró que, tanto estudiantes como profesores realizaron un gran esfuerzo de adaptación y, pese a las dificultades, fue posible alcanzar los resultados de aprendizaje establecidos en la guía docente de la asignatura. Como es habitual, no todo el alumnado se implicó del mismo modo, resultando una tasa de rendimiento del $75 \%$ (aprobados / presentados) similar a la de cursos anteriores. Sin embargo, la tasa de éxito (aprobados / presentados) fue del $100 \%$, mientras que en cursos anteriores estaba en_torno del $90 \%$, lo que atribuimos fundamentalmente a una mayor dedicación del alumnado.

Los nuevos materiales desarrollados, las nuevas tecnologías utilizadas, el uso de diferentes metodologías didácticas... abrieron un mundo de posibilidades sobre el que resultaba necesario reflexionar. Surgió una oportunidad de transformar progresivamente la docencia tradicionalmente impartida en un proceso más dinámico y activo, que incorporase diversas actividades para reforzar y completar las sesiones presenciales. La oportunidad de diseñar un proceso más motivador, que facilite además el seguimiento de la asignatura en aquellos casos en los que los alumnos que, por motivos justificados, no pudiesen asistir a clase en un modelo presencial.

\subsection{Reflexión y aprendizaje}

La experiencia vivida durante el confinamiento y la rápida adaptación a un modelo de docencia totalmente online permitió dar un salto en las metodologías docentes. Surgió la oportunidad de aprovechar los nuevos materiales desarrollados y el conocimiento adquirido para rediseñar el proceso de enseñanza - aprendizaje, dotándolo de muchos más recursos para que cualquier estudiante tuviese múltiples oportunidades de asimilar y poner en práctica los conocimientos adquiridos, de una forma activa, visual y motivadora. Un cambio progresivo 
y constante, impulsado por el apoyo a la formación del profesorado en nuevas tecnologías, pedagogía o metodologías docentes.

El objetivo ahora era desarrollar un nuevo modelo, presencial o semipresencial, que tratase de incrementar: motivación, implicación, aprendizaje activo, clase invertida (trabajar conceptos antes y aprovechar más las horas de clase) (Marqués, 2016), flexibilidad, disponibilidad de materiales para afianzar contenidos, autoevaluación. De igual modo, tratar que el nuevo modelo permitiese, cuando no fuese posible la presencia física del alumnado, comunicarse utilizando otros medios, como videoconferencia, correo electrónico o tutorías virtuales.

Para ello, resultaba necesario:

- Modificar los hábitos y rutinas tradicionalmente adquiridos.

- Rediseñar la docencia, para incorporar actividades a desarrollar antes, durante y después de las sesiones presenciales.

- Desarrollar materiales complementarios a los apuntes de la asignatura, que fuesen visuales y prácticos, capaces de enlazar información directamente accesible desde internet, como videos, documentos, esquemas, imágenes...

- Reemplazar las clases tradicionalmente expositivas por otras más dinámicas, con una participación más activa por parte del estudiantado.

- Apoyar a los estudiantes que, de forma justificada, no pudiesen asistir a clase.

\subsection{Formación del profesorado}

Tanto para adaptarse a una docencia $100 \%$ online como para rediseñar la docencia presencial o semipresencial, resultó fundamental mejorar la competencia virtual del profesorado. La universidad respondió de forma eficiente y puso a disposición de la comunidad universitaria multitud de cursos, diseñados de forma que pudiesen ser seguidos por el mayor número de usuarios posible, incluso en modalidad de autoformación. Así, se ofertaron diversos cursos específicos, flexibles, con materiales audiovisuales que permitieron recibir la formación al propio ritmo y según las posibilidades y disponibilidad particulares. $A$ modo de ejemplo, cabe citar que, en el caso concreto de la asignatura de Mdc3, el profesorado atendió cursos relacionados con las siguientes materias:

- Herramientas audiovisuales para la docencia online.

- Blended learning (Salinas, 2018).

- Diseño de actividades para la docencia y evaluación en línea e invertida.

- Clases dinámicas y participativas mediante el uso de herramientas de Google Workspace.

- $\quad$ Creación de contenido multimedia interactivo utilizando el módulo H5P del Aula Virtual.

- Diseño de cuestionarios mediante Aula Virtual. 


\subsection{Mejoras introducidas en la asignatura Materiales de Construcción III}

La formación recibida resultó esencial para mejorar la transmisión de conocimiento, rediseñar la docencia y facilitar al alumnado materiales que les permitan poner en práctica los conocimientos teóricos adquiridos, mejorando sus competencias y resultados de aprendizaje. Así, se diseñaron y desarrollaron diferentes tipos de actividades, a realizar:

- Antes de las prácticas de laboratorio: cuyo objetivo era que los alumnos revisaran y trabajaran los conceptos teóricos que serían posteriormente desarrollados en la sesión presencial.

- Durante las prácticas de laboratorio: Se rediseñó el trabajo a desarrollar, adaptándolo a las circunstancias sanitarias del momento y a las actividades a realizar antes y después.

- Después de las sesiones presenciales de teoría, problemas y laboratorios: se prepararon cuestionarios de autoevaluación a través del aula virtual, que les permitiesen comprobar el grado de conocimiento adquirido.

Las figuras 2 y 3 muestran ejemplos de actividades desarrolladas utilizando el módulo H5P (CEDEC, 2021), recientemente integrado en al aula virtual (AV). En la Universitat Jaume I el AV utiliza la plataforma moodle y, de forma sencilla e intuitiva, resulta un gran apoyo complementario a las sesiones presenciales. La incorporación del entorno de creación de contenido H5P resulta verdaderamente útil y abre un gran abanico de posibilidades para integrar actividades dinámicas, motivadoras e interactivas en el aula virtual. La presentación interactiva (figura 2) es una herramienta que permite combinar la presentación de información (mediante introducción de texto, enlaces a videos, figuras, páginas web...), con cuestiones de diversos tipos (respuesta múltiple, verdadero / falso, rellenar espacios en blanco (figura $2 a$ ), arrastrar palabras sobre huecos (figura $2 b$ )...), tratando así de fomentar una lectura activa y comprensiva.

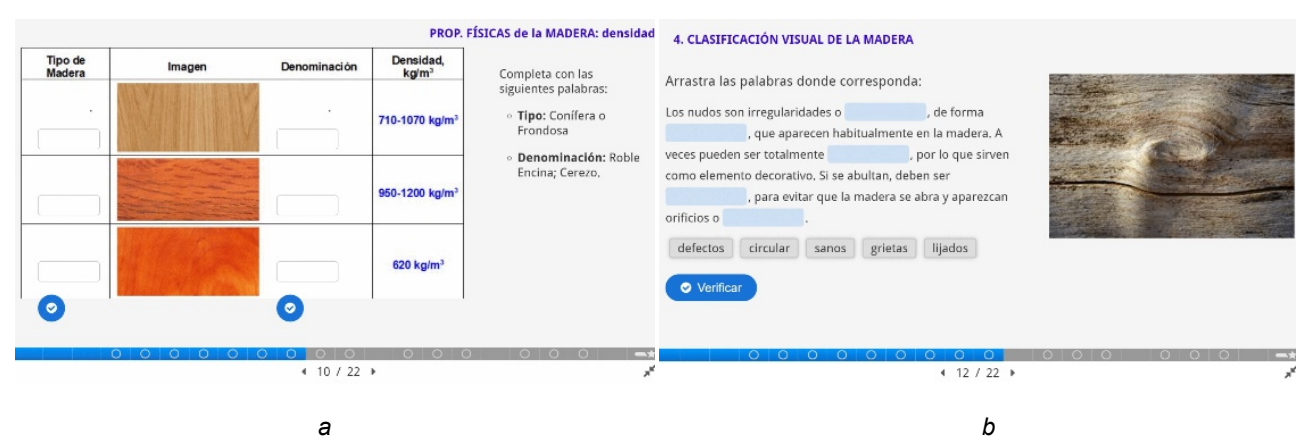

Fig. 2 Presentación interactiva diseñada con el módulo H5P para la asignatura Mdc3 
El video interactivo (figura 3) resulta también una herramienta excelente para completar las actividades presenciales pues, partiendo de un video (vinculado mediante enlace o desarrollado por nosotros a partir de fotos o video) permite intercalar actividades interactivas: como preguntas relacionadas con información que aparecerá en el video (figura 3a, pueden incorporarse antes o después de que aparezca dicha información), resumen del video a partir de cuestiones creadas por el profesorado (figura $3 b$ )...

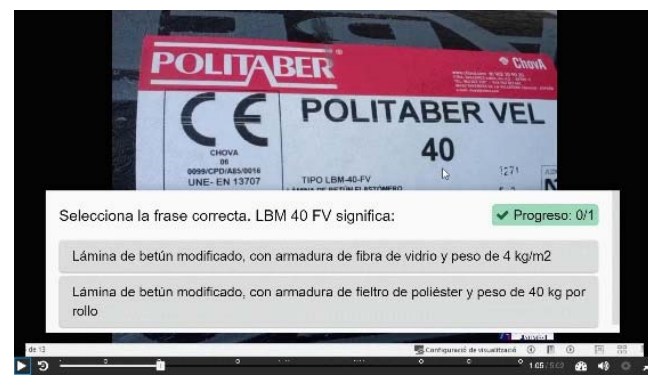

a

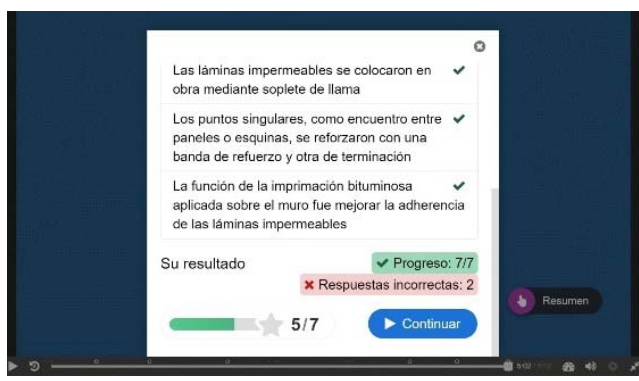

Fig. 3 Video interactivo diseñado con el módulo H5P para la asignatura Mdc3

La figura 4 presenta un cuestionario de autoevaluación propuesto para uno de los temas de la asignatura. El alumnado debe completarlo al finalizar el desarrollo del tema correspondiente, y les permite autoevaluar el nivel de conocimiento adquirido.
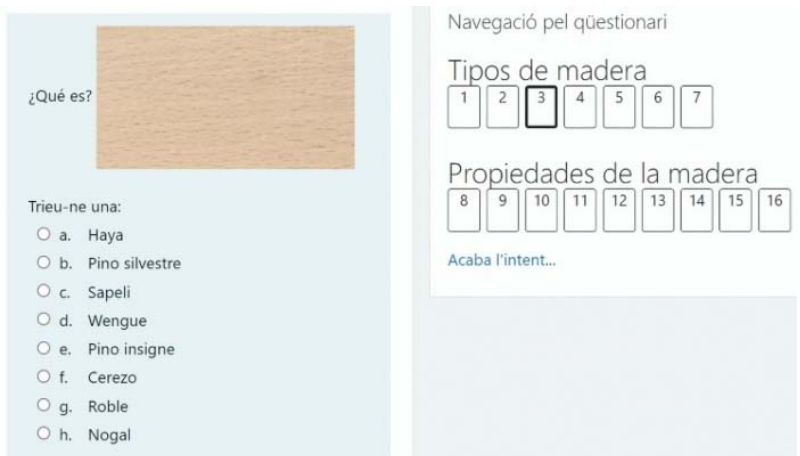

Fig. 4 Cuestionario de autoevaluación diseñado mediante el AV para la asignatura $M d c 3$

En definitiva, se ha tratado de aprovechar los materiales desarrollados y el conocimiento adquirido derivados de la situación sanitaria vivida para rediseñar la docencia y proponer actividades más atractivas, interactivas y motivadoras, que fomenten la participación e implicación del estudiantado en su propio proceso de aprendizaje. Combinar la docencia presencial con actividades online, de forma síncrona o asíncrona, permite reforzar y ampliar significativamente las oportunidades de las que dispone el alumnado para comprender y trabajar los conceptos teóricos, ponerlos en práctica y mejorar los resultados de aprendizaje. 


\subsection{Futuras propuestas de mejora}

Las diferentes actividades diseñadas para la asignatura Mdc3 han permitido una mayor implicación y motivación por parte del alumnado. No obstante, todavía queda mucho camino por recorrer:

- En ocasiones los estudiantes muestran poca disposición a realizar las actividades antes de la clase, pues no están habituados a ello. Introducir estas actividades de forma cada vez más sistemática les ayudaría a integrar una nueva forma de trabajo.

- Se pueden rediseñar más actividades complementarias, de refuerzo a las sesiones presenciales.

- Se pueden rediseñar más sesiones presenciales para invertir la clase (flipped clasroom), con el fin de aprovechar al máximo el tiempo presencial para el trabajo en actividades más complejas, como la resolución de problemas y ejercicios de aplicación práctica.

Los cambios propuestos pueden ser progresivos, pues el diseño de nuevas actividades complementarias y modificar las sesiones presenciales para invertir el orden de la clase requieren tiempo; además de un cambio de hábitos y rutinas, por parte de alumnado y profesores. No obstante, el esfuerzo vale la pena, pues una participación más activa del estudiantado en su propio proceso de aprendizaje derivará en una mayor motivación y un aprendizaje más duradero y significativo.

\section{Conclusiones}

La situación sobrevenida originada por la pandemia mundial de la COVID-19, la inseguridad y falta de comunicación presencial vividas durante el confinamiento, o la formación insuficiente en nuevas tecnologías generaron una fuerte necesidad y motivación. El esfuerzo realizado por la comunidad universitaria permitió transformar el proceso de enseñanzaaprendizaje, mejorándolo en muchos sentidos:

- Desde la universidad se ha dado un fuerte impulso a la formación del profesorado en nuevas tecnologías de la educación, con el desarrollo de guías, el apoyo de personal especializado, cursos de formación para el desarrollo de materiales multimedia o el uso de herramientas para la comunicación online.

- Gracias a una oferta de formación flexible, con materiales dinámicos y audiovisuales, el profesorado hemos podido mejorar significativamente nuestras competencias digitales.

- Fruto de la experiencia vivida, se ha logrado mejorar el proceso de enseñanzaaprendizaje, desarrollando nuevos materiales de apoyo y refuerzo de las actividades presenciales, que permiten y refuerzan el autoaprendizaje; así como facilitando el seguimiento de la asignatura cuando los alumnos no pueden asistir a las clases.

En definitiva, las dificultades vividas impulsaron un cambio hacia un proceso de enseñanzaaprendizaje más flexible, dinámico, activo y motivador; un proceso más centrado en el estudiantado. 


\section{Referencias}

ADELL SEGURA, J.; ANDRÉS CEBRIÁN, P. (2020). "Luces y sombras de la experiencia del confinamiento y el paso a la "Enseñanza en línea de emergencia» (CENT)". En IV Jornadas de innovación educativa DIMEU. Camino hacia la Virtualidad en la Universitat Jaume I. Castelló de la Plana: Publicacions de la Universitat Jaume I. Servei de Comunicació i Publicacions.

CEDEC, CENTRO NACIONAL DE DESARROLLO CURRICULAR EN SISTEMAS NO PROPIETARIOS. ¿Qué puede hacer H5p por mis alumnos?. < https://cedec.intef.es/que-puede-hacer-h5p-por-misalumnos/ > [Consulta: 13 de mayo de 2021] [personal o institucional]

CENT, CENTRE D'EDUCACIÓ I NOVES TECNOLOGIES DE LA UNIVERSITAT JAUME I. $<$ https://cent.uji.es/pub/> [Consulta: 13 de mayo de 2021]

MARQUÉS, M. (2016). "Qué hay detrás de la clase al revés (flipped classroom)". En Actas de las XXII Jornadas sobre la Enseñanza Universitaria de la Informática, 6-8 de julio, Almería. 77-84.

MARTÍNEZ-MOYA, J.A.; PITARCH-ROIG, A.; GARCÍA-ESPARZA, J.A.; RUÁ-AGUILAR, M.J. y CABEZA GONZÁLEZ, M. (2020) "Implementation of coordinated vertical project at technical architecture bachelor's degree, En ICERI 2020, 13th annual International Conference of Education, Research and Innovation, 9-10 November, Sevilla: IATED. 966-975.

PITARCH-ROIG, A.M.; MARTíNEZ-MOYÁ, J.A.; SÁEZ-RIQUELME, B.; REIG-CERDÁ, L.; MÁNEZPITARCH, M.J. "Analysis of non face-to-face teaching of the technical architecture bachelor's degree during the covid-19 state of alarm. Learned lessons." En ICERI 2020, 13th annual International Conference of Education, Research and Innovation, 9-10 November, Sevilla: IATED. 1785-1793.

REIG, L., RUÁ, M.J., BRAUliO, M., LeCHA, A., HUEDO, P. GALlego, T., PITARCH, A., SÁEZ, B., BABILONI, J. (2017). "Learning by doing in the bachelor's degree of technical architecture at the Universitat Jaume I". En EDULEARN 17, 9th annual International Conference on education and New Learning Technologies, 3-5 de julio, Barcelona: IATED. 7312-7321.

SALINAS, J., DE BENITO, B., PÉREZ, A., GISBERT, M. (2018). “¿Qué es el Blended Learning? Una revisión desde la investigación educativa" en RIED. Revista Iberoamericana de Educación a Distancia, 21, 1, 195-213. https://doi.org/10.5944/ried.21.1.18859. 